\title{
Radiation Induced Alopecia: An Under-Appreciated Side Effect of Whole Brain Radiotherapy and Strategies to Ameliorate it
}

\author{
Irfan Ahmad ${ }^{1 *}$, Kabir Sardana ${ }^{2}$, Kundan Singh Chufal $^{3}$ and Chandi Prasad Bhatt ${ }^{1}$
}

${ }^{1}$ Deptt of Radiotherapy, Batra Hospital \& Medical Research Centre, 1 Tughlakabad Institutional Area, New Delhi, India

${ }^{2}$ Deptt of Dermatology, Dr. Ram Manohar Lohia Hospital and Post Graduate Institute of Medical Education and Research, Baba Kharak Singh Marg, New Delhi, India

${ }^{3}$ Deptt of Radiotherapy, Rajiv Gandhi Cancer Institute \& Research Centre, Rohini, New Delhi, India

Keywords: Radiation; Alopecia; Whole brain radiotherapy

\section{Introduction}

Alopecia is considered an unavoidable consequence of successful cancer treatment and the mechanism of which is intertwined with the inherent nature of anti-cancer therapy, which targets rapidly proliferating cells. Hair loss is a precursor to loss of self-image in affected patients and can have a profound negative impact on patients [1]. These patients also report poorer quality of life, lower self-esteem and heightened self-consciousness [2,3]. While the importance of a successful oncological outcome cannot be over-emphasized, research focused on prevention of this distressing adverse effect is relatively sparse.

The propensity of anti-cancer treatment resulting in alopecia is different for each modality, with chemotherapy being most common due its systemic nature of administration [4]. With radiotherapy and its locally directed nature, alopecia occurs less frequently than chemotherapy overall, however it has a higher likelihood of resulting in permanent hair loss [5]. The most common radiotherapy treatment which causes alopecia is Whole Brain Radiotherapy (WBRT) which is used to manage brain metastases [6]. The clinical incidence of brain metastases in all patients diagnosed with cancer is $15-30 \%$ [6]. With the global incidence of cancer expected to reach 17.1 million cases/ year by the year 2020, the number of patients who will eventually receive this treatment and be exposed to this side effect is significant [7]. Another factor to consider is that with standardized surveillance protocols for non-central nervous system malignancies and sensitive neuroimaging modalities, brain metastases are being detected earlier and more frequently $[6,8]$. While the improvement in overall survival for these patients is debatable (due to a perceived lead-time bias), the length of time they will experience the side effects is not. It is also worth emphasizing that in recent years, the management of selected patients with a favorable prognostic profile has been shifting towards stereotactic radiosurgery (SRS) $[6,8]$. However, the reality of the disease process is that the most favorable prognostic subgroup constitutes only $9-16 \%$ of all patients with brain metastases, thereby limiting the widespread utilization of SRS [9].

Radiotherapy (RT) induced alopecia is a part of the spectrum of dose-dependent changes which occur when skin is irradiated and can be classified radiobiologically on the basis of reversibility (transient versus permanent) [5]. RT induces apoptosis in the actively dividing matrix cells in the hair bulb thereby interrupting the anagen phase of hair cycle and resulting in dystrophic anagen hair loss [10]. The onset is abrupt (within 1-3 weeks of treatment initiation), progression is rapid and diffusely affects the scalp hair [5,10]. Microscopic changes can be appreciated within 1-2 days of exposure represented by reduced mitotic index in matrix cells, shrinkage of hair bulb and reduced hair diameter $[11,12]$. The latency between exposure and hair loss corresponds to the period during which the matrix cells become progressively depleted. The dose threshold for this effect is extremely low, 0.75-2 Gray (Gy) delivered in a single treatment session [13]. Regrowth is possible as long as a few matrix cells survive along with adequate contact with dermal microvasculature and may occur between 3 weeks to 3 months after the completion of treatment $[11,12]$. However the probability of regrowth is inversely related to total delivered dose. Permanent alopecia occurs after a delivered dose of $7 \mathrm{~Gy}$ in a single session or after a total delivered dose of $43 \mathrm{~Gy}$ in a fractionated treatment regimen [corresponding equivalent dose in 2 Gy (EQD2) fractions with alpha/beta ratio of $2=36.9 \mathrm{~Gy}][13,14]$.

In clinical practice, WBRT regimens are fractionated and a relatively large single session dose of 7 Gy would be considered unacceptable $[6,8]$. The probability of developing permanent alopecia with the recommended regimens for WBRT (30 Gy in 10 fractions of $3 \mathrm{~Gy}$ each, over 2 weeks with EQD2=37.5 Gy; or 37.5 Gy in 15 fractions of $2.5 \mathrm{~Gy}$ each, over 3 weeks with EQD2=42.2 Gy) exceed 50\% [6,8,14]. From a practical standpoint, mitigation of transient alopecia by reducing dose delivered to scalp through the utilization of advanced RT delivery techniques may not be feasible, due to the extreme radio-sensitivity of germinal matrix cells. Consequently, clinical research utilizing novel pharmacological agents and advanced RT delivery techniques have focused on preventing permanent alopecia.

Pharmacologic interventions directed at reducing the incidence of RT induced alopecia has mostly been studied in pre-clinical animal models [15]. The only agent that has been evaluated clinically is Tempol (4-hydroxy-2,2,6,6-tetramethylpiperidin-1-oxyl), a member of the nitroxide group of chemical compounds. It acts as a radio-protector by exerting anti-oxidant activity and counteracting RT induced free radical damage (Figure 1) [15]. Besides being membrane-permeable, which favors topical application (thereby mitigating concerns with respect to systemic administration), it also has negligible systemic absorption [16]. A phase I trial performed on patients undergoing WBRT (30 Gy in 10 fractions of 3 Gy each, over 2 weeks) with topical Tempol application reported moderate protection from hair loss at 3 months, with a favorable toxicity profile [16]. Based on these results, a double blind, placebo controlled phase II trial (NCT00801086) was initiated by the same research group and the results are awaited.

Some novel research has also been recently reported which may

*Corresponding author: Irfan Ahmad, Deptt of Radiotherapy, Batra Hospital \& Medical Research Centre, 1 Tughlakabad Institutional Area, New Delhi, India, Tel: 0091-9953399688; Fax: 0091-11-2995 6255; E-mail: irfan.a@icloud.com

Received March 05, 2018; Accepted March 12, 2018; Published March 16, 2018

Citation: Ahmad I, Sardana K, Chufal KS, Bhatt CP (2018) Radiation Induced Alopecia: An Under-Appreciated Side Effect of Whole Brain Radiotherapy and Strategies to Ameliorate it. J Nucl Med Radiat Ther S9: 002. doi: 10.4172/2155 9619.S9-002

Copyright: $\odot 2018$ Ahmad I, et al. This is an open-access article distributed under the terms of the Creative Commons Attribution License, which permits unrestricted use, distribution, and reproduction in any medium, provided the original author and source are credited. 


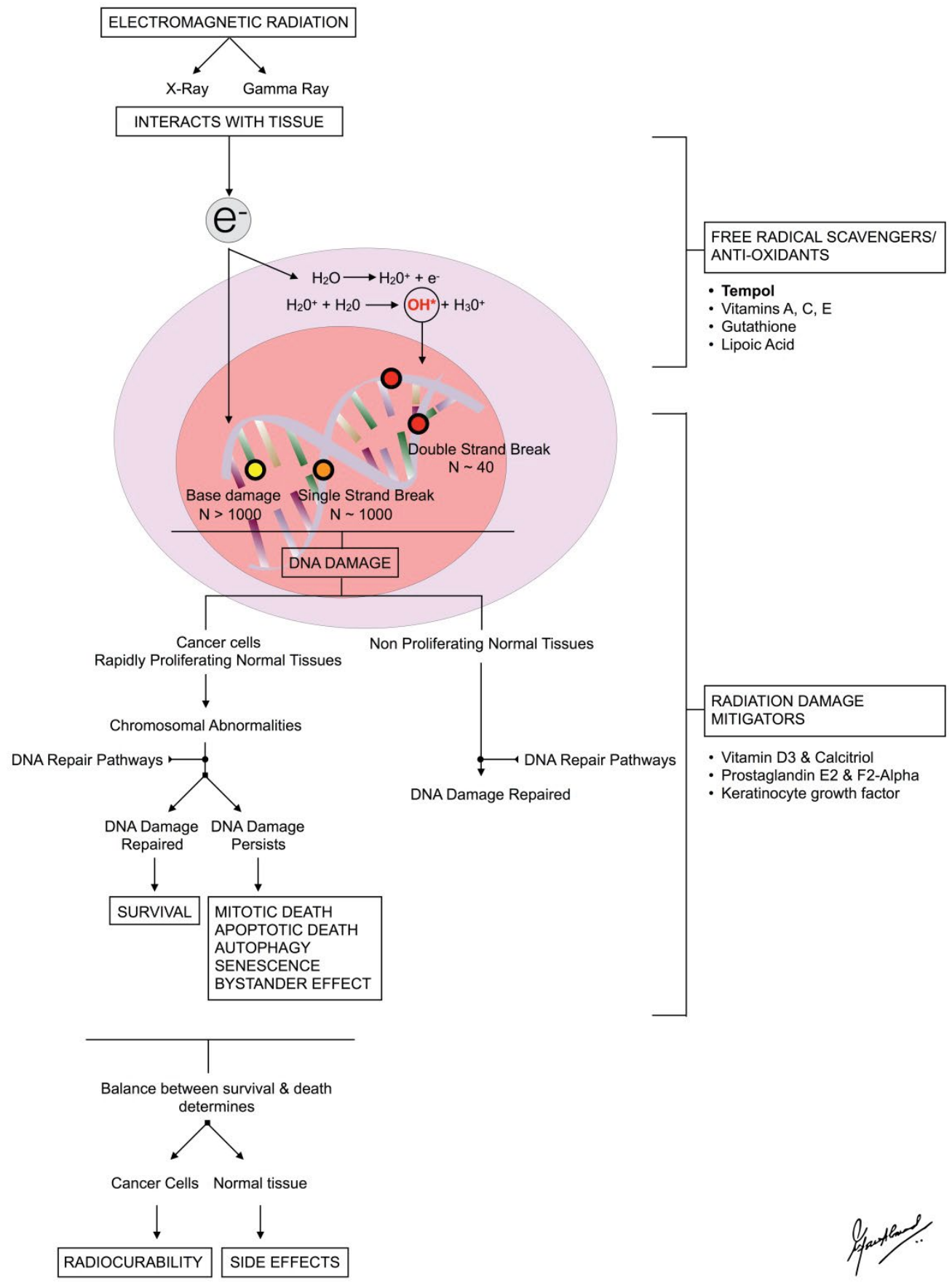

Figure 1: An overview of interaction of electromagnetic radiation with tissue and the resulting biologic effects. The pharmacologic agents which have been studied in preventing alopecia are also shown with their predominant mechanism of action.

yield novel agents in the future. In growing hair follicles (HF), quiescent stem cells (SC) are maintained in the bulge region, and hair bulbs at the base contain rapidly dividing, yet genotoxicity-sensitive transitamplifying cells (TAC) that maintain hair growth [17]. HFs mobilize ectopic progenitors from distinct TAC compartments for regeneration in adaptation to the severity of dystrophy induced by ionizing radiation (IR). Specifically, after low-dose IR, keratin 5+ basal hair bulb progenitors, rather than bulge SCs, are quickly activated to replenish matrix cells and regenerate all the layers of HFs, demonstrating their plasticity. After high-dose IR, when both matrix and hair bulb cells are depleted, the surviving outer root sheath cells rapidly acquired an SC-like state and fuel HF regeneration. Their progeny then homed back to SC niche, supporting new cycles of HF growth. The authors concluded that IR induces HF dystrophy along with hair loss, and suppressed WNT signalling in a p53- and dose-dependent manner [17]. Another study showed that mTORC1 signalling is activated 
Citation: Ahmad I, Sardana K, Chufal KS, Bhatt CP (2018) Radiation Induced Alopecia: An Under-Appreciated Side Effect of Whole Brain Radiotherapy and Strategies to Ameliorate it. J Nucl Med Radiat Ther S9: 002. doi: 10.4172/2155-9619.S9-002

Page 3 of 4

after irradiation and is required for timely regeneration of the TAC pool of hair follicles, so that hair growth can resume after radiation injury [18]. Fibroblast growth factor (FGF) 18 is strongly expressed in telogen HFs to maintain the telogen phase and it has now been shown that FGF18 is responsible for the radioresistance of telogen HFs, and can be a potential radioprotector [19]. Another potential agent could be 12-o-tetradecanoylphorbol-13-acetate (TPA), which accelerates re-entry of hair follicles into anagen phase. This drug activated AKT signalling in the epidermis, hair infundibulum, bulge and hair bulb, and WNT signalling after hair follicle stem cells proliferation [20].

Prior to discussing advanced RT delivery techniques, interested readers may wish to review Figure 2, which is a comparison of dose distributions achieved when using conventional radiotherapy and volumetric modulated arc therapy (VMAT) and the difference in deposited dose measured from the scalp surface to $5 \mathrm{~mm}$ depth. The potential for prevention of alopecia with these techniques has been
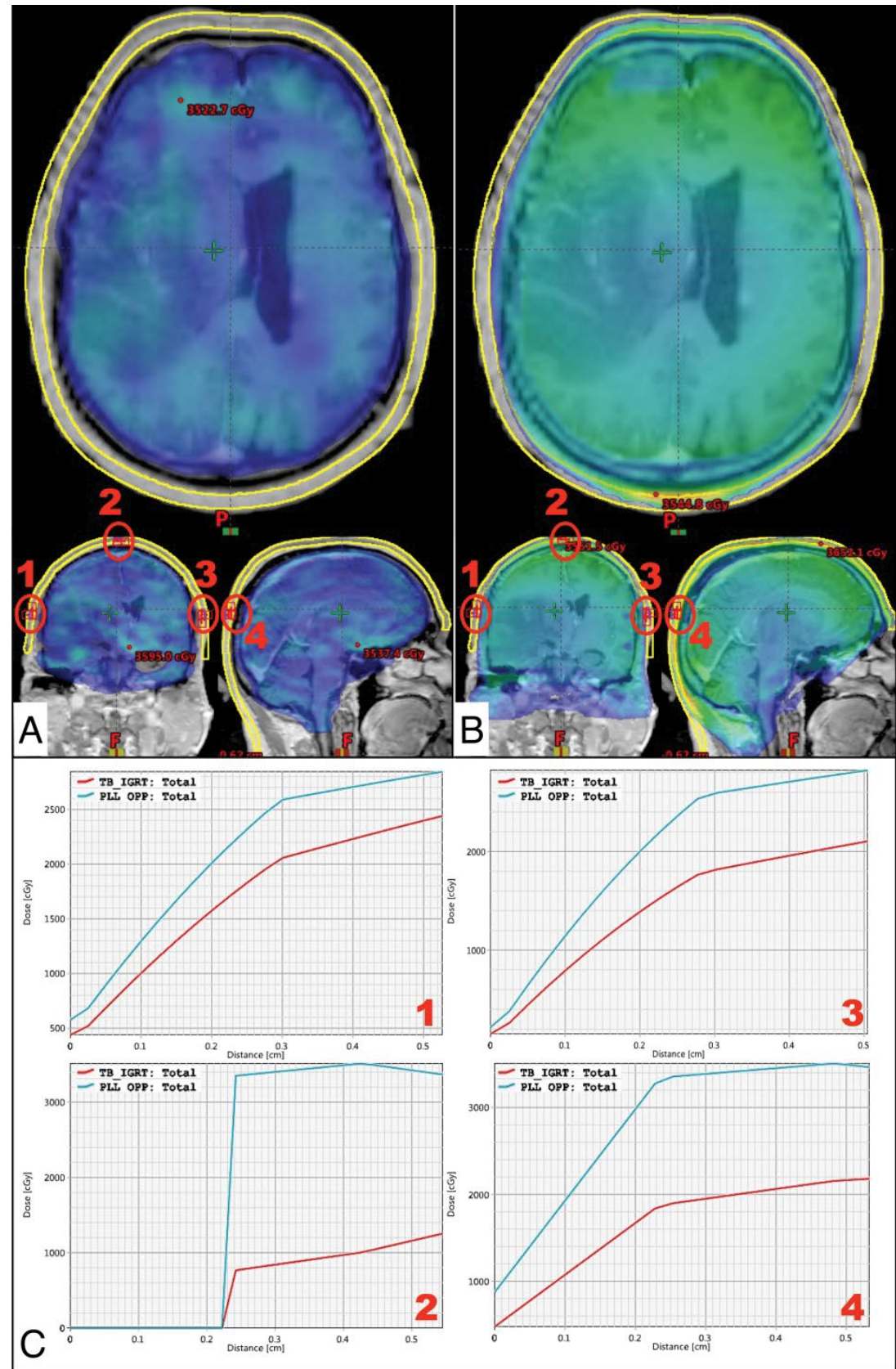

Figure 2: A comparison of WBRT dose distributions planned via VMAT and conventional parallel opposed techniques. (A) Dose distribution achieved with VMAT plan (clockwise from top, axial, sagittal and coronal planes). (B) Dose distribution achieved with conventional parallel opposed plan (clockwise from top, axial, sagittal and coronal planes). In both plans, four representative points (labeled 1, 2, 3, and 4 with red ovals) have been highlighted to measure the dose deposited from the surface to $5 \mathrm{~mm}$ depth from surface. (C) Dose profiles from surface of scalp to $5 \mathrm{~mm}$ depth at the four representative points shown in A and B, with dose in centigray (cGy) on Y-axis and depth from surface on X-axis. Note that for all measured points, the dose deposited at $4 \mathrm{~mm}$ to $5 \mathrm{~mm}$ depth is higher for conventional parallel opposed plan (PLL_OPP, Blue curve) than VMAT plan (TB_IGRT). 
Citation: Ahmad I, Sardana K, Chufal KS, Bhatt CP (2018) Radiation Induced Alopecia: An Under-Appreciated Side Effect of Whole Brain Radiotherapy and Strategies to Ameliorate it. J Nucl Med Radiat Ther S9: 002. doi: 10.4172/2155-9619.S9-002

demonstrated in dosimetric studies; however few clinical studies have been performed. A phase II trial was recently reported which explored the effect of sparing hair follicles during WBRT via VMAT, on alopecia-related quality of life (QoL) parameters and investigator based assessment of hair loss [21]. Ten patients were prospectively enrolled in this trial and received $20 \mathrm{~Gy}$ in 5 fractions over 1 week. Alopecia-related QoL parameters and hair loss assessment was performed one month after completion of RT. The authors reported no improvement in QoL scores pertaining to hair loss and reported $75 \%$ hair loss at one month post-completion of WBRT, which resulted in an early termination of the trial. However the chief criticism of this trial is the timing of data collection for hair loss, which overlaps with the period during which transient alopecia would manifest in patients, due to its low threshold dose. A few investigators have reported hair regrowth after transient alopecia, 3 to 6 months after completion of RT and rates of permanent alopecia ranging from $0 \%$ to $31 \%$ with the use of advanced RT delivery techniques $[22,23]$. It is also interesting to note that besides sparing the scalp, these techniques can be used for memory preservation (by sparing the hippocampus) and selectively increasing the delivered dose to the brain metastases to increase probability of local control (simultaneous integrated boost) [24,25]. Our group has recently reported on the combination of scalp and hippocampal sparing WBRT along with simultaneous integrated boost in the treatment of solitary brain metastasis, with full scalp hair regrowth at 2 months post WBRT, memory preservation and near complete response in the lesion [26]. Though these techniques are promising, level 1 recommendations for their routine use are lacking, for which well-designed randomized controlled trials are needed. It will also be of interest to evaluate whether a synergistic effect of scalp sparing RT techniques and topical tempol application exists.

In conclusion, radiation induced alopecia secondary to whole brain radiotherapy is a significant problem affecting a large population of cancer patients. Research on its prevention has focused on the use of radio-protectors or advanced RT delivery techniques to spare the scalp. Basic research has highlighted some novel pathways that could possibly circumvent RT induced alopecia including augmenting the WNT signalling, mTORC1 signalling pathway and possibly by up-regulating FGF 18. However, due to the low threshold of radiation dose which results in transient alopecia, efforts to prevent it may not be feasible and therefore the aim of future trials should be to minimize the rate of permanent alopecia.

\section{References}

1. Dorr VJ (1998) A practitioner's guide to cancer-related alopecia. Semin Onco 25: $562-570$

2. Williamson D, Gonzalez M, Finlay AY (2001) The effect of hair loss on quality of life. J Eur Acad Dermatol Venereol 15: 137-139.

3. Steinmann D, Paelecke-Habermann Y, Geinitz H, Aschoff R, Bayerl A, et al. (2012) Prospective evaluation of quality of life effects in patients undergoing palliative radiotherapy for brain metastases. BMC cancer 12: 283.

4. Karakunnel JJ, Qazi IM, Berger Am (2014) DeVita, Hellman, and Rosenberg's cancer: Principles \& practice of oncology. (10th edn), Wolters Kluwer Health/ Lippincott Williams \& Wilkins, Philadelphia

5. Kirkpatrick JP, Milano MT, Constine LS, Vujaskovic Z, Marks LB (2013) Perez $\&$ Brady's principles and practice of radiation oncology. (6th edn), Lippincott Williams \& Wilkins, Philadelphia.

6. Nichols EM, Patchell RA, Regine WF, Kwok Y (2013) Perez \& Brady's principles and practice of radiation oncology. (6th edn), Lippincott Williams \& Wilkins, Philadelphia.

7. Ferlay J, Soerjomataram I, Ervik M, Dikshit R, Eser S, et al. (2013) GLOBOCAN 2012 v1.0, Cancer Incidence and Mortality Worldwide. IARC CancerBase No.
11 [Internet]. International Agency for Research on Cancer, Lyon, France.

8. Suh JH, Chao ST, Peereboom DM, Barnett GH (2014) DeVita, Hellman, and Rosenberg's cancer: principles \& practice of oncology. (10th edn), Wolters Kluwer Health/ Lippincott Williams \& Wilkins, Philadelphia.

9. Nieder C, Mehta MP (2009) Prognostic indices for brain metastases-usefulness and challenges. Radiat Oncol 4: 10.

10. Pasch MC (2012) Kanerva's Occupational Dermatology. (2nd edn), SpringerVerlag, Berlin Heidelberg.

11. Malkinson FD, Keane JT (1981) Radiobiology of the skin: Review of some effects on epidermis and hair. $\mathrm{J}$ Invest Dermatol 77: 133-138.

12. Awwad HK (1990) Radiation oncology: Radiobiological and physiological perspectives: The boundary-zone between clinical radiotherapy and fundamental radiobiology and physiology. (1st edn), Springer, Dordrecht.

13. Hamilton CS, Potten CS, Denham JW, O'Brien PC, Kron T, et al. (1997) Response of human hair cortical cells to fractionated radiotherapy. Radiother Oncol 43: 289-292.

14. Lawenda BD, Gagne HM, Gierga DP, Niemierko A, Wong WM, et al. (2004) Permanent alopecia after cranial irradiation: Dose-response relationship. Int J Radiat Oncol Biol Phys 60: 879-887.

15. Sourati A, Ameri A, Malekzadeh M (2017) Acute side effects of radiation therapy. (1st edn), Springer, Cham.

16. Metz JM, Smith D, Mick R, Lustig R, Mitchell J, et al. (2004) A phase I study of topical tempol for the prevention of alopecia induced by whole brain radiotherapy. Clin Cancer Res 10: 6411-6417.

17. Huang WY, Lai SF, Chiu HY, Chang M, Plikus MV, et al. (2017) Mobilizing transit-amplifying cell-derived ectopic progenitors prevents hair loss from chemotherapy or radiation therapy. Cancer Res 77: 6083-6096.

18. Wang WH, Chien TH, Fan SM, Huang WY, Lai SF, et al. (2017) Activation of mTORC1 signaling is required for timely hair follicle regeneration from radiation injury. Radiation Res 188: 681-689.

19. Kawano M, Umeda S, Yasuda T, Fujita M, Ishikawa A, et al. (2016) FGF18 signaling in the hair cycle resting phase determines radioresistance of hair follicles by arresting hair cycling. Adv Radiat Oncol 1: 170-181.

20. Quu W, Lei M, Zhou L, Bai X, Lai X, et al. (2017) Hair follicle stem cell proliferation, Akt and Wnt signaling activation in TPA-induced hair regeneration. Histochem Cell Biol 147: 749-758.

21. De Puysseleyr A, Van De Velde J, Speleers B, Vercauteren T, Goedgebeur A, et al. (2014) Hair-sparing whole brain radiotherapy with volumetric arc therapy in patients treated for brain metastases: Dosimetric and clinical results of a phase II trial. Radiat Oncol 9: 170.

22. Weber DC, Caparrotti F, Laouiti M, Malek K (2011) Simultaneous in-field boost for patients with 1 to 4 brain metastasis/es treated with volumetric modulated arc therapy: A prospective study on quality-of-life. Radiat Oncol 6: 79

23. Kao J, Darakchiev B, Conboy L, Ogurek S, Sharma N, et al. (2015) Tumo directed, scalp sparing intensity modulated whole brain radiotherapy for brain metastases. Technol Cancer Res Treat 14: 547-555.

24. Gondi V, Pugh SL, Tome WA, Caine C, Corn B, et al. (2014) Preservation of memory with conformal avoidance of the hippocampal neural stem-cell compartment during whole-brain radiotherapy for brain metastases (RTOG 0933): A phase II multi-institutional trial. J Clin Oncol 32: 3810-3816.

25. Ferro M, Chiesa S, Macchia G, Cilla S, Bertini F, et al. (2017) Intensity modulated radiation therapy with simultaneous integrated boost in patients with brain oligometastases: A phase 1 study (ISIDE-BM-1). Int J Radiat Oncol Biol Phys 97: 82-90.

26. Ahmad I, Chufal KS, Bhatt CP, Rathour S (2017) Treating patients with brain metastases has evolved: Scalp-sparing, hippocampal avoidance whole brain radiotherapy with simultaneous integrated boost. BMJ Case Rep, 2017.

This article was originally published in a special issue, Radiation Oncology and Radiobiology handled by Editor(s). Richard Maximus Fleming, The Camelot Foundation, United States 